\title{
Observations Concerning the Mass Variation in a Galilean - Type Relativity
}

\author{
Marius Arghirescu \\ State Office for Inventions and Trademarks, Bucharest, Romania
}

\section{Email address:}

arghirescu.marius@osim.ro,maris3a@yahoo.com

\section{To cite this article:}

Marius Arghirescu. Observations Concerning the Mass Variation in a Galilean - Type Relativity. International Journal of High Energy Physics. Vol. 5, No. 1, 2018, pp. 44-54. doi: 10.11648/j.ijhep.20180501.15

Received: May 2, 2018; Accepted: May 17, 2018; Published: June 11, 2018

\begin{abstract}
By an electric charge model of static type, with spherical distribution of field quanta, periodically emitted, and by the Galilean relativity, there are re-obtained the Lorentz's expressions of the speed-depending longitudinal and transversal mass of a charged particle accelerated by a quanta flux pressure, as apparent effect generated by a real decreasing of the values of longitudinal and transversal electric field, $\mathrm{E}_{\mathrm{L}} \sim \gamma^{-3} ; \mathrm{E}_{\mathrm{T}} \sim \gamma^{-1},\left(\gamma=1 / \sqrt{ }\left(1-\mathrm{v}^{2} / \mathrm{c}^{2}\right)\right.$, being re-obtained also the general form of the Doppler-Fizeau effect. The invariance of the Lorentz force expression indicates a relativist variation of the magnetic field induction, in the form: $\mathrm{B} \sim \gamma^{-1}$, which explains the experimental result of the Kaufmann-Bucherer experiments. It is proposed a classical expression of mass and charge variation, in the form: $\mathrm{m}=\mathrm{m}_{\mathrm{o}} / \alpha ; \mathrm{q}=\mathrm{q}_{\mathrm{o}} / \alpha,\left(\alpha=\left(1-\mathrm{v}^{2} / 2 \mathrm{c}^{2}\right)\right)$, which may results as real variation by a significant density of a super-fluid medium of the quantum vacuum containing etherons $\left(\mathrm{m}_{\mathrm{g}, \mathrm{s}}=10^{-60} \div 10^{-70} \mathrm{~kg}\right)$ and quantons $\left(\mathrm{m}_{\mathrm{h}}=\mathrm{h} \cdot 1 / \mathrm{c}^{2}=7.37 \times 10^{-51} \mathrm{~kg}\right)$, by an relativist etherono-quantonic vortex $\Gamma_{\mathrm{r}}(\mathrm{v})$ which is added to the similar vortex $\Gamma_{\mu}(\mathrm{v})$ of the particle's magnetic moment which is increased with the speed, if the particle's spin is rectangular to its impulse. The explicative model may explain also the photon's energy in correlation with its electromagnetic properties.
\end{abstract}

Keywords: Relativist Mass Variation, Einstein's Relativity, Doppler-Fizeau Effect, Kaufmann-Bucherer Experiment, Photon Rest Mass

\section{Introduction}

It is known that the special Einsteinian relativity generated controversies about the validity of the light speed constancy postulate, resulted mainly from the so-called "twin paradox" generated by the Einsteinian relation of time dilation and from the Einsteinian mass variation with its speed which. Even if initially this phenomenon resulted as an observational effect generated by the limitation of the speed of light quanta used for observing the phenomenon, it induced subsequently the conclusion of a real mass increase at relativistic particle's speed.

In the same time, for consistency with the photon's properties, it resulted the concept of 'zero rest mass' of quanta having the speed $\mathrm{v}_{\mathrm{c}}=\mathrm{c}$-conclusion generalized also for the case of other bosons (graviton, gluon), although there are experiments that indicated a photon's behavior similar to those of other particles and implicitly - the need to consider a non-null rest mass, like in the case of the "stopped light" experiment [1], which showed a considerable reduction of the photon's velocity at the passing through a Bose-Einstein condensate, and the experiment of the Bose-Einstein condensate of photons obtaining [2].

There are also theoretical arguments that highlights the need to consider a non-null rest mass of photon [3] and of other bosons such as the gluon of strong interaction, as arguments in this sense being the hard gamma-quantum conversion into a negatron-positron pair $(\mathrm{e}+-\mathrm{e}-)$ at the passing through the field of an atomic nucleus and the fact that the gluonic shell of the quarks contributes to the restmass of the formed mesonic or baryonic particles.

In the context of relativist quantum mechanics, it seems that the standard model of elementary particles cannot explain the rest mass of relativistic particles without the Higgs mechanism of rest mass generation through the Higgs boson field of the quantum vacuum, although there are some attempts to explain the observed phenomenology without this theoretical consideration [4].

The tendency to interpret the Einsteinian relation of speeddepending mass variation as a real phenomenon is also 
criticized by some authors [5], in the context in which even Einstein mentioned that his theory of special relativity yields conclusions in the Known Universe, which is the result of the Real Universe observing with finite speed rays. There are known attempts to avoid the postulate of light speed constancy, proposing, for example, an exponential relation of mass variation with its speed, [6], At the same time, the evidence of field-like properties of the dark energy [7] that determines the expansion of the Universe, in connection with the experimental evidences of the quantum vacuum 'zero point energy', such as the Casimir effect, reintroduced the old concept of 'ether' into the phenomenology of particles and of fields phenomena but also in some theories of quantum type, although in the same time the special Einsteinian relativitybased on the exclusion of the ether concept -as consequence of the Michelson-Morley's experiment result, is maintained.

- It may be concluded in this case that - at least partially, the solution to the problem of naturally describing of the microcosm phenomenology implies the need to return to a Galilean relativity applied in an Euclidean space.

$$
\mathrm{E}=\mathrm{mc}^{2}=\mathrm{m}_{0} \mathrm{c}^{2}+1 / 2 \mathrm{mv}^{2}=\mathrm{E}_{0}+\mathrm{E}_{\mathrm{c}} ;
$$

which gives the solution: $\mathrm{m}=\mathrm{m}_{\mathrm{o}} / \alpha=\mathrm{m}_{\mathrm{o}} /\left(1-\mathrm{v}^{2} / 2 \mathrm{c}^{2}\right)$.

By replacing in eqn. (2) the total energy and the impulse with the corresponding operators of the quantum mechanics, is obtained the Schrödinger equation for the non-relativist case.

Let us consider - according to a Galilean-type relativity, the validity of this relation (2) for the whole range of speeds, including for $\mathrm{v}=\mathrm{c}$, thus applicable also to photons, which- in this case are "non- privileged" particles, i.e.- without the
The paper presents a phenomenological description of some relativistic effects in the dynamics of charged elementary particles, on the basis of a Galilean relativity.

\section{The Solving of Particles Mass Variation Problem in the Galilean Relativity}

As it is well known, the specific form of the mass-energy conservation law in the Einstein's relativity, compatible with the principle of metric's invariance, has the form:

$$
E^{2}=\left(m c^{2}\right)^{2}=\left(m_{0} c^{2}\right)^{2}+p^{2} c^{2} ; m=m_{o} \gamma=m_{o} / \sqrt{ }\left(1-v^{2} / c^{2}\right)
$$

and gives the solution: $\mathrm{m}=\mathrm{m}_{\mathrm{o}} \cdot \gamma$ with $\gamma=1 / \sqrt{ }\left(1-\mathrm{v}^{2} / \mathrm{c}^{2}\right)$.

The only form of the energy conservation law included in eqn. (1) that avoids the conclusion of particle's mass growth to infinity with its velocity, is those of the classical limit ( $\mathrm{v}$ $<<$ ):

$$
\mathrm{m}=\mathrm{m}_{\mathrm{o}} / \alpha=\mathrm{m}_{\mathrm{o}} /\left(1-\mathrm{v}^{2} / 2 \mathrm{c}^{2}\right)
$$

hypothesis of a null rest-mass of photon.

However, the question arises: how may be explained the relation describing the acceleration ' $a$ ' of an electrically charged particle, given by an electric force $F_{a}=m \cdot a=q \cdot E$, which indicates- by the Einsteinian relation (1), the possibility of particles accelerating (including also electrons) until energies of the order of some $\mathrm{GeV}$ or even $\mathrm{TeV}$ ?

To find a compatibility of the classic relation:

$$
F_{a}^{e}=\mathrm{m} \cdot \mathrm{a}_{\mathrm{q}}=q \cdot E ; \quad \mathrm{m}=\mathrm{m}_{0} / \alpha ; \quad \alpha=\left(1-\mathrm{v}^{2} / 2 c^{2}\right)
$$

with the relation of Einsteinian type:

$$
F_{a}^{e}=\mathrm{m}^{\prime} \cdot \mathrm{a}_{\mathrm{q}}=q^{\prime} \cdot E^{\prime} ; \quad \mathrm{m}^{\prime}=\mathrm{m}_{0} \cdot \gamma ; \quad \gamma=1 / \sqrt{\left(1-\mathrm{v}^{2} / c^{2}\right)}
$$

for which we consider as correct only the acceleration's expression, we suppose- for instance, a relativistic variation also for the q-charge and/or the electric E- field and we will analyze in which physical conditions the two relations (3) and (4) gives the same value or close values for the acceleration $\mathrm{a}_{\mathrm{q}}$, i.e.:

$$
a_{q}=\mathrm{dv} / \mathrm{dt}=\mathrm{q} \cdot \mathrm{E} / \mathrm{m} \approx \mathrm{q}^{\prime} \cdot \mathrm{E}^{\prime} / \mathrm{m}^{\prime}
$$

For a plausible answer to this question through the classic relation (3), the following observations must be made:

- In the Einsteinian relativity, the electric charge and the longitudinal component, parallel to the particle's speed, of the accelerating E-field, are considered invariants when we pass from a fixed reference system $\mathrm{O}_{\mathrm{s}}$ to an inertial system, $\mathrm{O}_{\mathrm{i}}$, i.e.: $\mathrm{q}\left(\mathrm{v}_{\mathrm{e}}\right)=\mathrm{q}(0) ; \mathrm{E}_{\|}\left(\mathrm{v}_{\mathrm{e}}\right)=\mathrm{E}_{\|}(0)$, although the charge's density is considered variable with the charge's speed: $\rho_{\mathrm{e}}\left(\mathrm{v}_{\mathrm{e}}\right)$ $=\rho_{\mathrm{e}}(0) \cdot \gamma$, by considering a relativistic contraction of the charge's volume with its speed: $\vartheta_{\mathrm{q}}\left(\mathrm{v}_{\mathrm{e}}\right)=\vartheta_{\mathrm{q}}(0) / \gamma$.

These considerations - although consistent with experimental observations specific to the electrodynamics such as the Kaufmann-Bucherer-Neumann experiment [8], do not take into account the microphysical mechanism of the electric field and the electric charge generation.

According to a cold genesis theory of fields (CGT $[9,10]$ ), the electric field $\mathrm{E}$ is explained by the existence of a spherically-symmetric flow of vectorial photons of the accelerating Q-charge's field, ("vectons" - in CGT), with the impulse density: $p_{v}(0)=\rho_{\mathrm{v}} c-$ for a static interaction between $\mathrm{Q}$ and $\mathrm{q}\left(\right.$ if $\mathrm{v}_{\mathrm{e}}=0$ ) and with an expression of the E- field of the form:

$$
E(r .0)=\mathrm{k}_{1} \cdot \rho_{\mathrm{v}} \mathrm{v}_{\mathrm{v}}^{2} \quad \text { with } \quad \mathrm{v}_{\mathrm{v}}=\left(\mathrm{c} \pm \mathrm{v}_{\mathrm{e}}\right) ; \mathrm{k}_{1}=4 \pi \cdot \mathrm{a}^{2} / e ; \quad(\mathrm{a}=1.41 \mathrm{fm})
$$


i.e.- dependent on the $\mathrm{v}_{\mathrm{v}}$ - speed of quanta relative to the interaction semi-surface: $\mathrm{S}_{\mathrm{x}}=2 \pi \mathrm{r}_{0}{ }^{2}=\mathrm{n} \cdot 2 \pi \mathrm{a}^{2}=\mathrm{nS}_{\mathrm{x}}{ }^{0}$, of the charge $\mathrm{q}=\mathrm{n} \cdot \mathrm{e}$. In CGT, $\mathrm{S}_{\mathrm{x}}{ }^{0}$ is considered as being the interaction section of the electron with the E-field quanta: $S_{x}{ }^{0}$ $=\pi\left(\mathrm{a}+\mathrm{r}_{\mathrm{v}}\right)^{2}$, with: $\mathrm{a}=1,41 \mathrm{fm}$ - the radius of an electron with the e-charge on its surface and $r_{v}=0,41 \cdot a-$ the gauge radius of the vecton, (CGT), [10].

The accelerating force $F_{e}$, given by repulsion between a charge $Q$ with the mass $M$ and a charge $q$ with the mass $m_{q}$, results- in this case, (according to CGT), from the impulse variation of the field quanta at the quasi-elastic collision with the semi-surface $S_{x}=2 \pi r^{2}$, i.e:

$$
F_{r}(\mathrm{q})=\mathrm{S}_{\mathrm{x}} \frac{\Delta\left(p_{c}\right)_{r}}{\Delta t}=\mathrm{S}_{\mathrm{x}} \frac{2\left(\rho_{s} \Delta x \cdot \mathrm{v}_{\mathrm{v}}\right)_{r}}{\Delta t}=S^{0} \rho_{v} \cdot \mathrm{v}_{\mathrm{v}}^{2}=q \cdot E_{r}(0) ; \quad \mathrm{q}=\frac{S^{0}}{k_{1}} ; \quad \mathrm{S}^{0}=4 \pi \cdot r^{2}=\mathrm{n} \cdot 4 \pi \cdot \mathrm{a}^{2}
$$

Also, in CGT, the expression of the magnetic induction results in the form:

$$
\mathrm{B}(\mathrm{r})=\mathrm{k}_{1} \cdot \rho_{c} \mathrm{v}_{\mathrm{v}} ; \quad \mathrm{v}_{\mathrm{v}} \approx c
$$

the magnetic moment of particles resulting in CGT from an etherono-quantonic vortex of primordial dark energy: $\Gamma_{\mu}=$ $\Gamma_{\mathrm{A}}+\Gamma_{\mathrm{B}}$, formed by a component $\Gamma_{\mathrm{A}}$ of "heavy" etherons $\left(\mathrm{m}_{\mathrm{s}}\right.$ $\approx 10^{-60} \mathrm{~kg} / \mathrm{m}^{3}$ ), explaining physically the magnetic potential A and a component of "quantons" $\left(\mathrm{m}_{\mathrm{h}}=\mathrm{hv} / \mathrm{c}^{2}=\mathrm{h} \cdot 1 / \mathrm{c}^{2}\right.$ $=7.37 \times 10^{-51} \mathrm{~kg} / \mathrm{m}^{3}$ ), explaining physically the magnetic induction $\mathrm{B},[9,10]$.

-The conversion of eqn. (7) into a equation of the gravitational force or of an etheronic drag force at the $\mathrm{m}_{\mathrm{q}}$ mass deplacing is obtained in CGT by the relations: $\mathrm{k}_{1}=$ $\left(\mathrm{m}_{\mathrm{e}} / \mathrm{e}\right) \cdot \mathrm{k}_{\mathrm{h}} ; \mathrm{q}=\mathrm{q}_{\mathrm{g}}=\left(\mathrm{e} / \mathrm{m}_{\mathrm{e}}\right) \cdot \mathrm{m}_{\mathrm{q}}$, in which $\mathrm{k}_{\mathrm{h}}=\mathrm{S}_{\mathrm{h}} / 2 \mathrm{~m}_{\mathrm{h}}=27.4$; $\left(\mathrm{S}_{\mathrm{h}}=4 \pi \mathrm{r}_{\mathrm{h}}{ }^{2}\right.$-the quanton's surface).

-The quarks confinement force is explained in CGT by a "bag" model given by the static quantum pressure gradient of the scalar charge of impenetrable quantum volume of the nucleon, [6], which explains also its repulsive property, observed in scattering interactions.

According to eqn. (7) it results as plausible a phenomenological decrease of the accelerating field $\mathrm{E}(\mathrm{v})$ which determines the acceleration of the particle $\mathrm{m}(\mathrm{q}, \mathrm{v})$, in its inertial coordinate system $\mathrm{O}_{\mathrm{i}}$, as consequence of the quanta speed decreasing relative to the accelerated charge, $\mathrm{q}$.

From a phenomenological point of view, it results in consequence that the Einsteinian relation (4) could be approximated by the classical relation (3) by considering a

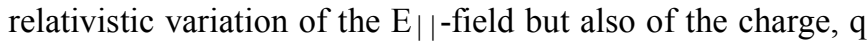
(v), according to a relation of the form:

$$
\begin{gathered}
\mathrm{m}(\mathrm{v}) \cdot \mathrm{a}_{\mathrm{q}}=\mathrm{q}(\mathrm{v}) \cdot \mathrm{E}_{\mid}(\mathrm{v}) ; \mathrm{m}=\mathrm{m}_{\mathrm{o}} / \alpha^{\prime} \\
\mathrm{q}=\mathrm{q}_{\mathrm{o}} / \alpha^{\prime} \alpha=\left(1-\mathrm{v}^{2} / 2 \mathrm{c}^{2}\right)
\end{gathered}
$$

The equality: $a_{\mathrm{q}}(v, \alpha)=a_{\mathrm{q}}(\mathrm{v}, \gamma)$ may results- in this case, from a variation of the E-field, of the form: $E_{||}(v)=E_{0} / \gamma$, by considering the same relativistic variation of the q-charge and of the mass, i.e.: $\mathrm{m}(\mathrm{v}) / \mathrm{m}_{0}=\mathrm{q}(\mathrm{v}) / \mathrm{q}_{0}$.

The increase of the mass and of the charge with the speed through the factor $\alpha$ could result as real only if the increased values: $\mathrm{m}(\mathrm{v}), \mathrm{q}(\mathrm{v})$ could be evidenced as such also in the inertial system $\mathrm{O}_{i}$ of the $\mathrm{m}$ - particle and if exists an energy: $\mathrm{E}=\Delta \mathrm{m} \cdot \mathrm{c}^{2}$ which can explain the forming of the additional mass $\Delta$ m, (i.e.-a vortexial energy, given by vacuum quanta).

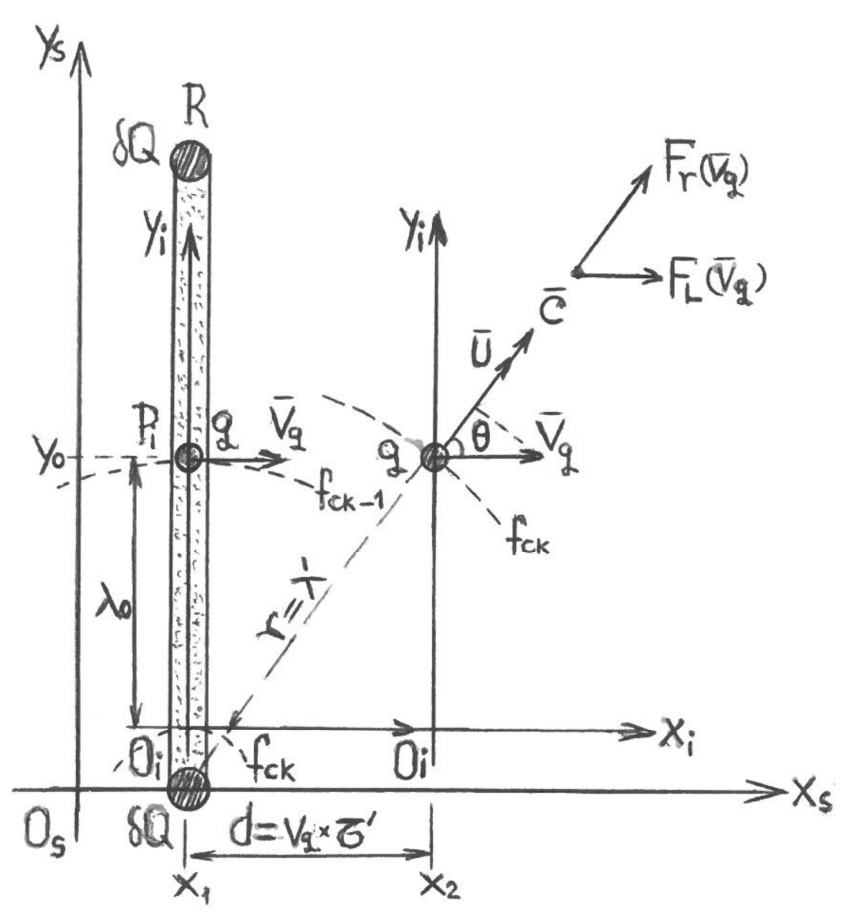

Figure 1. The relativist generating of the accelerating $\boldsymbol{E}$-field.

For analyze the variation of the accelerating electric Efield at a relativistic speed of the q-charge, generated by the relativistic variation of the impulse density: $\rho_{\mathrm{v}} \mathrm{v}$ of field quanta, we will consider that a $\mathrm{m}_{\mathrm{q}}$-particle with the electric charge $\mathrm{q}$ is accelerated inside a linear accelerator by the accelerating electrodes $\mathrm{R}$ of ring form, and that the q-charge is- at the time $t_{0}=0$, in a point $P_{i}\left(0, y_{0}\right)$ at the center of the accelerating electrode $\mathrm{R}$ which generates an electric $\mathrm{E}$ - field through its electric charge $\mathrm{Q}$, the q-charge and the attached inertial reference system $\mathrm{O}_{i}$ having - in the reference system $\mathrm{O}_{\mathrm{S}}$, the speed $\mathrm{v}_{\mathrm{q}}$-considered as quasi-constant within a sufficiently short period of time, $\delta$ t, (Figure 1).

We may consider also that an electric E- field is produced by a small charge' part $\delta \mathrm{Q}$ of the R-electrode, according to the equations (6) and (7), but by fronts $f_{q}$ of vectorial quanta ("vectons") of impulse density:

$$
\mathrm{pv}=\rho \mathrm{vc}=(1 / \lambda 0) \cdot \rho \mathrm{sc} ; \quad \lambda 0=\mathrm{c} \cdot \tau 0
$$

having the speed $\mathrm{v}_{\mathrm{v}}=\mathrm{c}$ in the reference system $\mathrm{O}_{\mathrm{S}}$ and $\mathrm{a}$ surface density of quanta: $\rho_{\mathrm{s}}$ in the $\mathrm{f}_{\mathrm{q}}$ - fronts- spaced at intervals $\lambda_{\mathrm{o}}=\mathrm{c} \cdot \tau_{0}=\mathrm{y}_{0}$. We consider also that at the moment $\mathrm{t}$ 
$=0 \mathrm{a} \mathrm{f}_{\mathrm{ck}}$-front of vectons passes through the origin 0 of the inertial system $\mathrm{O}_{\mathrm{i}}$ while the front $\mathrm{f}_{\text {ck-1 }}$ strikes the q-charge with the speed: $v_{v}=c$, (figure 1).

This $\mathrm{f}_{\mathrm{ck}}$-front of quanta will hit the surface of the q-charge rectangular to the direction ' $r$ ' (given by the position of qcharge in $\mathrm{O}_{\mathrm{S}}$ ) at a relative speed: $\mathrm{u}_{\mathrm{c}}=\mathrm{c}-\mathrm{v}_{\mathrm{e}}=\mathrm{c}-\mathrm{v}_{\mathrm{q}} \cdot \cos \theta$, $\left(\mathrm{v}_{\mathrm{q}}\right.$-the charge's speed) after a period of time $\tau^{\prime}$ in which the $f_{\mathrm{ck}}$-front of quanta cover the distance: $r=\lambda^{\prime}=\mathrm{c} \cdot \tau^{\prime}$ and the inertial reference system $\mathrm{O}_{\mathrm{i}}$ cover the distance: $\mathrm{d}=\mathrm{v}_{\mathrm{q}} \cdot \tau^{\prime}$, resulting that: $\mathrm{r}^{2}=\mathrm{d}^{2}+\mathrm{y}_{0}{ }^{2} \Rightarrow \tau^{\prime}=\tau_{0} \cdot \sqrt{ }\left(1-\mathrm{v}_{\mathrm{q}}{ }^{2} / \mathrm{c}^{2}\right)$.

Considering (CGT) that the electrical force is given by the quanta impulse variation per unit time, corresponding to a (quasi) elastic collision, because that the period $\tau^{\prime}$ results from the geometric relation: $\lambda^{, 2}-\mathrm{d}^{2}=\lambda_{0}^{2}$ in the form: $\tau^{\prime}=$ $\tau_{0} / \sqrt{ }\left(1-\mathrm{v}_{\mathrm{q}}^{2} / \mathrm{c}^{2}\right)$, it results- for the electric accelerating force $\mathrm{F}_{\mathrm{r}}\left(\mathrm{v}_{\mathrm{q}}\right)$ acting over the $\mathrm{q}$-charge, the relation:

$$
F_{r}(\mathrm{v})=\mathrm{S}_{\mathrm{x}} \frac{\Delta\left(p_{c}\right)_{r}}{\Delta t}=\mathrm{S}_{\mathrm{x}} \frac{2\left(\rho_{s} u_{c}\right)}{\tau^{\prime}}=S^{0} \rho_{s} \cdot\left(c-\mathrm{v}_{\mathrm{q}} \cos \theta\right) \frac{\sqrt{1-\frac{\mathrm{v}_{\mathrm{q}}^{2}}{\mathrm{c}^{2}}}}{\tau_{0}}=S^{0} \rho_{s} c \cdot\left(1-\frac{\mathrm{v}_{\mathrm{q}}^{2}}{\mathrm{c}^{2}}\right) \frac{\sqrt{1-\frac{\mathrm{v}_{\mathrm{q}}^{2}}{\mathrm{c}^{2}}}}{\tau_{0}}=F_{r}(0) \cdot\left(\sqrt{1-\frac{\mathrm{v}_{\mathrm{q}}^{2}}{\mathrm{c}^{2}}}\right)^{3}
$$

The correspondence with the form (6), (7) of the $E_{r}$-field and $F_{r}$-force is given by eq. (10).

The component in the longitudinal direction of this electric force is:

$$
F_{L}(\mathrm{v})=F_{r}(0) \cdot \cos \theta \cdot\left(\sqrt{1-\frac{\mathrm{v}_{\mathrm{q}}^{2}}{\mathrm{c}^{2}}}\right)^{3}=F_{L}(0) \cdot\left(\sqrt{1-\frac{\mathrm{v}_{\mathrm{q}}^{2}}{\mathrm{c}^{2}}}\right)^{3}
$$

in which: $F_{L}(0)$ represents the longitudinal component of the $F_{r}$-force generated by the charge $\delta Q$ and acting over a q-charge stationary in the system $\mathrm{O}_{\mathrm{s}}$ in the same point $\mathrm{P}_{\mathrm{j}}\left(\mathrm{d}, \mathrm{y}_{0}\right)$ as the accelerated $\mathrm{q}$-charge after a time $\delta \mathrm{t}=\tau$ '. By introducing the expression (12) in the relation (9), it results:

$$
a_{L}(\mathrm{v})=\frac{F_{L}(0)}{m_{q}} \cdot\left(\sqrt{1-\frac{\mathrm{v}_{\mathrm{q}}^{2}}{\mathrm{c}^{2}}}\right)^{3}=\frac{q}{m_{q}} E_{L}(0) \cdot\left(\sqrt{1-\frac{\mathrm{v}_{\mathrm{q}}^{2}}{\mathrm{c}^{2}}}\right)^{3}=\frac{q^{0}}{m_{q}^{0}} E_{L}(\mathrm{v})
$$

The relation (13), in the relativist Lorentz-Einstein hypothesis: $\mathrm{E}_{||}\left(\mathrm{v}_{\mathrm{e}}\right)=\mathrm{E}||(0) ; \mathrm{q}(\mathrm{v})=\mathrm{q}(0)$, suggests a variation of the $\mathrm{m}_{\mathrm{q}}$-mass with the speed in the longitudinal direction, of the form: $m_{L}(v)=m(0) \cdot \gamma^{3}$, which was theoretically deduced by Lorentz in 1904 and by Einstein in 1905- by the postulate of the light speed constancy, [11]. According to equations (11) - (13), it results only an apparent mass variation, in the form (4), the real variation with the charge's speed being those of the electric E-field in the frame of the inertial system $\mathrm{O}_{\mathrm{i}}$ of the accelerated q-charge.

The einsteinian form $(4): \mathrm{m}_{\mathrm{L}}(\mathrm{v})=\mathrm{m}(0) \cdot \gamma$ corresponds according also to eqn. (11), to the lorentzian form of the transversal electromagnetic mass [12] and to the postulate of the photon's speed constancy in vacuum, $(u=c)$, specific to the einsteinian relativity- but which is paradoxical for the longitudinal mass or E-field.

In the case of a q-charge accelerated by attraction, by an opposite $\delta \mathrm{Q}$-charge of an R-electrode, the relation (13) is maintained- according to the CGT model of electrical interaction, according to which the attractive interaction between two $q_{1}, q_{2}$ charges of opposed sign takes place by attraction between vectorial photons of opposed spins and opposed pseudo-charges in the inter-charges space, with the reduction of quantum pressure on the semi-surface $S_{x}$ corresponding to the space between them, the coulombian attractive force resulting from the mean value of the pressure: $\mathrm{P}_{\mathrm{v}}=\rho_{\mathrm{v}} \mathrm{w}^{2}(\mathrm{w} \approx \mathrm{c}$-for pseudo-stationary charges) of pseudoscalar photons of the quantum vacuum (vecton-antivecton pairs) acting on the opposed semi-surfaces, $S / / S_{x}$.

If the attracted $\mathrm{q}$-charge is moving at a $\mathrm{v}$-speed relative to the attracting $\delta \mathrm{Q}$-charge of the R-electrode, because that in this case we have: $\mathrm{w} \approx \mathrm{c}-\mathrm{v}_{\mathrm{e}}$, by repeating the reasoning specific to the case of charge accelerating by repulsion, we retrieve the relation (11).

The expression (13) of the longitudinal mass variation with the speed was deduced also by the Lorentzian model of electron's electromagnetic mass, from the electromagnetic moment variation: $\mathrm{P}_{\mathrm{EM}}=\int \varepsilon_{0} \mathrm{ExB} \cdot \mathrm{d}^{3} \mathrm{x}$, introduced by Abraham, which generates the force produced by the electron's self-field: $F_{\mathrm{SELF}}=\int \rho_{\mathrm{e}}(\mathrm{E}+\mathrm{vxB}) \cdot \mathrm{d}^{3} \mathrm{x}=-\mathrm{dP}_{\mathrm{EM}} / \mathrm{dt}$, [13].

It can be explained- in this case, by the relations (11)- (13), avoiding the paradox of infinite increasing of particle's mass by a relativist speed $\mathrm{v} \rightarrow \mathrm{c}$, the result of some experiments of accelerated electrons speed determining, (SLAC experiment, Brown et al., 1973 [14], Guiragossián et al., 1974 [15]), which showed the lack of difference between the speed of accelerated electrons at energies of 11-20.5 GeV and of some gamma radiation quanta of $15 \mathrm{GeV}$, (values corresponding to the Einsteinian relativist variation of mass).

It may be observed also that the Lorentzian relativist expression of mass variation with the speed in the transverse direction: $\mathrm{m}_{\mathrm{T}}(\mathrm{v})=\mathrm{m}(0) \cdot \gamma$, corresponds- by the relations (11) and (13), to a real variation of the accelerating E-field produced by the annular $\mathrm{R}$-electrode at the surface of the qcharge, in the form: $E_{r}(v)=E_{r}(0) / \gamma$ corresponding to the case 
in which the quanta of the photonic fronts $f_{c}$ hits the semisurface $S_{x}$ of the q-charge with the speed $v_{v}=c$ but with a frequency: $v\left(v_{\mathrm{q}}\right)=v_{0}(0) / \gamma$ - resulted from the variation of the period $\tau=1 / v$ and corresponding to the transversal Doppler effect.

-The correspondence with the Doppler-Fizeau effect of the relation (11) results from the following reasoning:
If- in the previous case, we replace the charge $\delta Q$ with a source of laser radiation with a frequency $v_{0}=\mathrm{c} / \lambda_{0}$ and in the inertial system $\mathrm{O}_{\mathrm{i}}$ we replace in the position $\mathrm{P}_{\mathrm{i}}\left(0, \mathrm{y}_{0}\right)$ the qcharge with an observer $A_{i}$, repeating the reasoning used for the obtaining of relation (11) will results that the observer $A_{i}$ will observe the laser radiation as having a modified wavelength: $\lambda_{\mathrm{s}}$.

$$
\lambda_{\mathrm{s}}=\mathrm{u} \cdot \tau^{\prime}=\mathrm{u} \cdot \tau_{0} \cdot \gamma=(\mathrm{u} / \mathrm{c}) \cdot \lambda_{0} \cdot \gamma ; \quad \gamma=1 / \sqrt{\left(1-\mathrm{v}^{2} / \mathrm{c}^{2}\right)} \quad ; \quad \lambda_{0}=\mathrm{c} \cdot \tau_{0}
$$

in which $u_{v}$ is the speed of radiation quanta relative to the observer $A_{i}$, which depends on the relative speed of the observer: $\mathrm{v}_{\mathrm{q}}|| \mathrm{x}$ according to the relation:

$$
\mathrm{u}_{\mathrm{v}}=\mathrm{c}-\mathrm{v}_{\mathrm{q}} \cdot \cos \theta=\mathrm{c}+\mathrm{v}_{\mathrm{s}} \cdot \cos \theta,
$$

in which: $v_{s}=-v_{q}$ is the speed of the laser source relative to the observer $A_{i}$. It is obtained in this case- by eqns. (14) and (15), the expression of the relativist wavelength $\lambda_{\mathrm{s}}$ corresponding to the general form of the Doppler-Fizeau effect [16]:

$$
\lambda_{s}=\lambda_{0} \cdot\left(1+\frac{\mathrm{v}_{\mathrm{s}}}{c} \cos \theta\right) / \sqrt{1-\frac{\mathrm{v}_{\mathrm{s}}^{2}}{\mathrm{c}^{2}}} ; \quad \theta=\pi / 2 \Rightarrow \lambda_{s}=\lambda_{0} / \sqrt{1-\frac{\mathrm{v}_{\mathrm{s}}^{2}}{\mathrm{c}^{2}}}
$$

which for $\theta=0$ gives the longitudinal $D-F$ effect and for $\theta=\pi / 2$ gives the transverse D-F effect.

In the case of a non-relativistic speeds: $\mathrm{v}_{\mathrm{q}}<<\mathrm{c}$ we have $\alpha \rightarrow 1$ and: $\mathrm{F}_{\mathrm{L}}(\mathrm{v}) \rightarrow \mathrm{F}_{\mathrm{L}}(0)=\mathrm{F}_{\mathrm{r}}(0) \cdot \cos \theta$.

Because that on the direction $\mathbf{r}$ the $E_{r}$-field quanta have the speed $v=c$, considering a small interval: $\delta r=n_{r} \lambda_{c}=n_{r} \cdot c \cdot \tau_{0}$, with a linear density $n_{0}=1 / \lambda_{0}$ of quanta fronts $f_{c}$, with the relations (6) and (11) of CGT, we have:

$$
F_{L}(\mathrm{v})=F_{r}(0) \cdot \cos \theta=\mathrm{S}_{\mathrm{x}} \frac{\delta\left(p_{c}\right)_{r}}{\delta t} \cdot \cos \theta=\mathrm{S}_{\mathrm{x}} \frac{2\left(\rho_{s} u_{c}\right)}{\tau} \cdot \cos \quad \theta=S^{0} \rho_{s} \cdot \frac{1}{\tau_{0} c} \cdot c^{2} \cdot \cos \quad \theta \approx S^{0} \rho_{v} \cdot c^{2} \cos \quad \theta=q \cdot E_{L}^{0}
$$

in which: $\mathrm{S}^{0}=2 \mathrm{~S}_{\mathrm{x}}=4 \pi \mathrm{r}_{\mathrm{o}}{ }^{2}=\mathrm{n} \cdot 4 \pi \mathrm{a}^{2},(\mathrm{n}=\mathrm{q} / \mathrm{e})$ and $\tau_{0} \mathrm{c}=\lambda_{0}$, in accordance with the basic eqn. (7).

If the q-charge has the $\mathrm{v}_{\mathrm{q}}$-speed rectangular to a homogenous $\mathrm{E}_{\mathrm{T}}$-field, we may obtain the expression of the transversal $\mathrm{E}_{\mathrm{T}}-$ field re-writing the equation for the $\mathrm{F}_{\mathrm{r}}(\mathrm{v})$ force in the form:

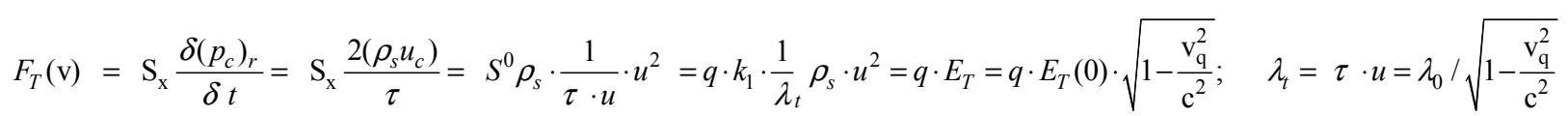

because that from eq. (15) and (16), for $\theta=\pi / 2, \mathrm{E}_{\mathrm{r}}(0)=\mathrm{E}_{\mathrm{T}}(0)$ it results that: $\mathrm{u}=\mathrm{c}$ and with $\tau=\tau_{0} \cdot \gamma, \Rightarrow \mathrm{E}_{\mathrm{T}}(\mathrm{v})=\mathrm{E}_{\mathrm{T}}(0) / \gamma$.

The acceleration of the q-charge resulted from the action of the transversal field $\mathrm{E}_{\mathrm{T}}(\mathrm{v})$ is:

$$
a_{T}(\mathrm{v})=\frac{F_{T}(0)}{m_{q}} \cdot\left(\sqrt{1-\frac{\mathrm{v}_{\mathrm{q}}^{2}}{\mathrm{c}^{2}}}\right)=\frac{q}{m_{q}} E_{T}(0) \cdot\left(\sqrt{1-\frac{\mathrm{v}_{\mathrm{q}}^{2}}{\mathrm{c}^{2}}}\right)=\frac{q^{0}}{m_{q}^{0}} E_{T}(\mathrm{v})
$$

relation which- in the relativist Lorentz-Einstein hypothesis: $q(v)=q(0)$, suggested a relativist variation of the $m_{q}-m_{a s s}$ corresponding to a transversal mass of the form: $\mathrm{m}_{\mathrm{L}}(\mathrm{v})=\mathrm{m}(0) \cdot \gamma$. Also, putting $\theta=0$ in eqn. (15) and (16) and replacing $\mathrm{u}, \lambda_{\mathrm{t}}$ in (18) with the obtained $\lambda_{1}$ and $u_{v}$ we find the same form for $E_{1}$ as those from eq. (11) in which $\theta=0$ : $E_{1}=E_{1}^{0}\left(1-v_{q} / c\right) \sqrt{ }(1-$ $\left.\mathrm{v}_{\mathrm{q}}^{2} / \mathrm{c}^{2}\right)$.

Comparing with the relativist Joules-Bernoulli equations [17] based on the Einsteinian relativity:

$$
E_{\perp}^{\prime}(\mathrm{v})=\gamma \cdot\left(E_{\perp}^{0}+\overrightarrow{\mathrm{v} x} \overrightarrow{\mathrm{B}}\right) ; \quad \mathrm{B}_{\perp}^{\prime}(\mathrm{v})=\gamma \cdot\left(B_{\perp}^{0}-\frac{1}{c^{2}} \overrightarrow{\mathrm{v} x} \overrightarrow{\mathrm{E}}\right) ; \quad \gamma=\left(1-\mathrm{v}^{2} / c^{2}\right)^{-\frac{1}{2}}
$$

which cannot be explained physically by eqn. (11), it results from eqns. (18), (19) and from the galilean invariance of the Lorentz force expression $\left(E_{L}=v \times B\right)$ that the general form of the fields $E_{\perp}$ ' $(v)$ and $B_{\perp}{ }^{\prime}(v)$, in the inertial system $O_{i}$ of the $q$ (v)-charge, must be the form: 


$$
E_{\perp}^{\prime}(\mathrm{v})=\gamma^{-1} \cdot\left(E_{\perp}^{0}+\overrightarrow{\mathrm{v} x} \overrightarrow{\mathrm{B}}\right) ; \quad \mathrm{B}_{\perp}^{\prime}(\mathrm{v})=\gamma^{-1} \cdot\left(B_{\perp}^{0}-\frac{1}{c^{2}} \overrightarrow{\mathrm{v} x} \overrightarrow{\mathrm{E}}\right) ; \quad \gamma=\left(1-\mathrm{v}^{2} / c^{2}\right)^{-\frac{1}{2}}
$$

It may be observed that the relativist variation of $\mathrm{B}_{\perp}$ '(v) conform to eqn. (21), resulted by the invariance of the physics laws:

$$
\mathrm{B}_{\perp}{ }^{\prime}(\mathrm{v})=\mathrm{E}_{\mathrm{L}}{ }^{\prime}(\mathrm{v}) / \mathrm{v}=\mathrm{B}_{\perp}{ }^{0} \cdot \gamma^{-1} ; \quad\left(\mathrm{E}_{\mathrm{L}}{ }^{\prime}(\mathrm{v})=\mathrm{E}_{\mathrm{L}}{ }^{0} \cdot \gamma^{-1}=\left(\mathrm{v} \mathrm{x} \mathrm{B} \mathrm{B}_{0}\right) \cdot \gamma^{-1}\right)
$$

may explain the result of the Kaufmann's [8] and of the Bucherer's experiments [18], (re-confirmed by Rogers et al. [19]), according to the next equations:

a) In the case of Kaufmann's experiment, consisting in the applying of an electric and magnetic fields aligned parallel to each other so that the deflections $y$ and $\mathrm{z}$ caused by them to electrons of beta-rays $(\mathrm{v} / \mathrm{x} ; \mathrm{v} \geq 0.9 \mathrm{c})$ were perpendicular to each other, using also the eqns. (21), we have:

$$
z^{2} / y=\left(\frac{\mathrm{eB}_{0} \mathrm{~b}}{\gamma \cdot \mathrm{m}_{\mathrm{e}} \mathrm{v}}\right)^{2} /\left(\frac{e E_{0} a}{\gamma \cdot \mathrm{m}_{\mathrm{e}} \mathrm{v}^{2}}\right)=\left(\frac{e}{\gamma \cdot m_{e}}\right) \cdot\left(\frac{\left(\mathrm{B}_{0} \mathrm{~b}\right)^{2}}{E_{0} a}\right) ; \quad \gamma=\left(1-\mathrm{v}^{2} / c^{2}\right)^{-\frac{1}{2}}
$$

with: a, b- constants specific to the experimental installation. It is retrieved by eqn. (22) the experimental result of the Kaufmann's experiment, which was considered as being given by the einsteinian speed-depending mass variation.

b) In the case of the Bucherer's experiment, consisting in the passing through a velocity filter using rectangulary $\mathrm{E}_{0}-\mathrm{B}^{0}$ fields, of some beta rays whose $v$-speed was such that the action of the electric $\mathrm{E}_{0}$-field and the magnetic $\mathrm{B}_{1}{ }^{0}$-field exactly compensated each other and thereafter- through a constant magnetic field $\mathrm{B}_{2}{ }^{0}$ which generates a deflection of radius $\mathrm{R}$, using also the eqns. (21) we have:

$$
\mathrm{v}=\left(\frac{\mathrm{e} \mathrm{E}_{0} \gamma^{-1}}{e B_{1}^{0} \gamma^{-1}}\right)=\frac{\mathrm{E}_{0}}{\mathrm{~B}_{1}^{0}}=\frac{e \cdot\left(B_{2}^{0} \cdot \gamma^{-1}\right)}{\mathrm{m}_{\mathrm{e}} R^{-1}} ; \quad \gamma=\left(1-\mathrm{v}^{2} / c^{2}\right)^{-\frac{1}{2}}
$$

It is retrieved by eqn. (23) the experimental result of the Bucherer's experiment, which was considered as being given by the Einsteinian speed-depending mass variation.

The possibility to explain the previous experiments by a variation of E- and B- fields of the form (21) was considered as Galilean alternative for the Kaufmann-Bucherer experiment explaining also in the book: „A new light in physics" (2017, [20]), but without explanations regarding the cause of the speed- depending variation of the $\mathrm{E}_{\mathrm{T}^{-}}$and $\mathrm{B}$ fields.

It may be mentioned also that the charge's variation with the speed was considered also by Asif Laghari [21] but in the form: $\mathrm{q}=\mathrm{q}_{0} \cdot \gamma$, by the Einsteinian relativity.

The question that arises is: if the speed-depending variation of both the mass $\mathrm{m}_{\mathrm{q}}$ and the charge $\mathrm{q}$ in the classic form (9) may be real or results only as formal variations.

\section{The Physical Possibility of a Real Mass Variation with the Speed}

In the context of admitting the relations (9) and (13), it is necessary to explain in which way the considered variation with the factor $1 / \alpha$ of the mass $m_{q}$ and of the charge $q$ could result as real, in the sense of the effect's existence also in the inertial system of the accelerated $\mathrm{m}_{\mathrm{q}}$ - particle.

A real growth of $\mathrm{m}_{\mathrm{q}^{-}}$and $\mathrm{q}-$ values with the particle's speed until a maximum value- double than the initial one, would be possible -according to CGT, by the generation of a relativistic etherono-quantonic vortex: $\Gamma_{\mathrm{r}}(\mathrm{v})=2 \pi \mathrm{r}_{\mu} \cdot \mathrm{v}_{\mathrm{e}}$ whichat values $\mathrm{v}_{\mathrm{e}} \rightarrow \mathrm{c}$ of the particle's speed, may increase the quanta density and the intrinsic energy of the magnetic moment's vortex of the q-charge: $\Gamma_{\mu}(\mathrm{v})=2 \pi \mathrm{r}_{\mu} \cdot \mathrm{c},\left(\mathrm{r}_{\mu}=\lambda / 2 \pi=\right.$ $\hbar / m_{\lambda} c$-the Compton radius ), but also its value and the spin's value, if $\mathrm{r}_{\mu}(\mathrm{v})=\mathrm{r}_{\mu}{ }^{0}=\hbar / \mathrm{m}_{0} \mathrm{c}$, according to the equation:

$$
\mu(\mathrm{v})=1 / 2 \mathrm{q}(\mathrm{v}) \cdot \mathrm{c} \cdot \mathrm{r}_{\mu}=\mu_{0} / \alpha=(\mathrm{q} / \mathrm{m})_{\mathrm{v}} \mathrm{S}_{\mathrm{q}} ;
$$

The microphysical dependence of the value of an elementary charge $\mathrm{q}=\mathrm{e}$ on the density of vectons and quantons of the electric and magnetic fields: $\rho_{v}, \rho_{c}$, at the echarge's surface of radius $r_{q}=a$, results in CGT through the eqns (6) and (8) which gives the equality:

$$
\rho_{v}(a)=\rho_{c}(a) \text { : }
$$

$$
\mathrm{E}(\mathrm{q}, \mathrm{a})=\mathrm{c} \cdot \mathrm{B}(\mathrm{q}, \mathrm{a}) ; \Rightarrow \mathrm{B}=\mathrm{k}_{1} \rho_{\mathrm{c}}(\mathrm{a}) \mathrm{c}=\mathrm{k}_{1} \rho_{\mathrm{v}}(\mathrm{a}) \mathrm{c} \sim \mu_{\mathrm{e}} \sim \mathrm{e} ;
$$

the link with the mass resulting-for the electron, from the relations $[9,10]$ :

$$
\mathrm{me}=2 \pi \mathrm{a} 3 \rho \mathrm{v}(\mathrm{a}) \sim \mathrm{e} ; \quad \mathrm{e}=4 \pi \mathrm{a} 2 / \mathrm{k} 1=4 \pi \mathrm{a} 2 \mathrm{k} 1 \varepsilon 0 \rho \mathrm{v}(\mathrm{a}) \mathrm{c} 2 ; \quad(\rho \mathrm{v}(\mathrm{a})=\mu 0 / \mathrm{k} 12)
$$

The conclusion of the magnetic moment's increase with the e-charge's speed allows the conclusion of a real increase of the $\mathrm{m}_{\mathrm{e}^{-}}$mass with its speed by the attracting of "naked photons" (limited to their inertial mass) from the quantum 
vacuum, vortexially confined and included in the particle's quantum volume by the attraction force generated by quantum potential of Bohm type, (of particle interaction with the sub-quantum medium), considered of quantum-vortexial nature also in other theories [22].

According to CGT $[9,10]$, the magnetic vortex: $\Gamma_{\mu}(0)=$ $2 \pi r_{\mu} \cdot c$, for the quasi-stationary particle, results by the interaction of omnidirectional etherono-quantonic winds of mean speed $v_{c}=c$ (formed by etherons $\left(\mathrm{m}_{\mathrm{g}, \mathrm{s}}=10^{-60} \div 10^{-70} \mathrm{~kg}\right.$ ) and by quantons $\left(\mathrm{m}_{\mathrm{h}}=\mathrm{h} \cdot 1 / \mathrm{c}^{2}=7.37 \times 10^{-51} \mathrm{~kg}\right)$ ) with the chiral super-dense kernel $\mathrm{m}_{0}$ of the e-charge.

Also, the relativist vortex: $\Gamma_{\mathrm{r}}(\mathrm{v})=2 \pi \mathrm{r}_{\mu} \cdot \mathrm{v}_{\mathrm{e}}$ results from the interaction of the pseudo-stationary etherono-quantonic medium of the quantum vacuum with the chiral super-dense $\mathrm{m}_{0}$-kernel of the charge $\mathrm{q}=\mathrm{e}$ having the $\mathrm{v}_{\mathrm{e}}$-speed and generates a supplementary self-potential $V_{\Gamma}(r)$ of the displaced particle, of Eulerian form, generated by the relativistic quanta impulse density: $\mathrm{p}_{\mathrm{s}}(\mathrm{r})=\rho_{\mathrm{s}}(\mathrm{r}) \cdot \mathrm{v}_{\mathrm{e}}$ and acting over the impenetrable quantum volume $v_{i}$ of an external particle, $[9,10,27]$, i.e.:

$$
V_{\Gamma}(r)=\mathrm{V}_{\Gamma}^{0} \cdot|\Psi|^{2} ; \quad \mathrm{V}_{\Gamma}^{0}=\frac{\vartheta_{1}}{2} \rho_{s}^{0} \mathrm{v}_{\mathrm{e}}^{2} ; \quad|\Psi|^{2}=\mathrm{R}^{2}=\mathrm{e}^{-\frac{\mathrm{r}}{\eta}} ; \Psi=\mathrm{R} \cdot \mathrm{e}^{-\mathrm{i} \frac{\mathrm{S}}{\hbar}} ; \mathrm{S}=v_{\mathrm{i}}\left(\rho_{s} \mathrm{v}_{\mathrm{e}}\right)_{r} \cdot l_{x} ; l_{\mathrm{x}} \perp \mathrm{r}
$$

It results that- for a real doubling of the $m_{e}-$ mass and of the q-charge's value at a speed $\mathrm{v}_{\mathrm{e}} \approx \mathrm{c}$, it is necessary that the density $\rho_{\mathrm{s}}^{0}$ of the pseudo-stationary etherono-quantonic medium of the quantum vacuum to be equal to the density $\rho_{c}{ }^{0}$ of the etherono-quantonic vortex $\Gamma_{\mu}(0)$. However, this effect would produce a quantum drag force $F_{r}(v)$ that would generates an apparent speed -depending mass increase effect and a particle's speed reducing, in contradiction with the first principle of the Newtonian dynamics.

This contradiction can be avoided by the d'Alembert's paradox [23], which shows that in the case of a fluid medium with zero or almost zero viscosity such as a superfluid, the drag force is also null. This conclusion is consistent with the fact that- according also to other etheronic theories, the subquantum medium must be considered an ideal fluid to which the laws of ideal super-fluids may be applied and with almost null viscosity [24], being consistent also with the arguments against the "tyred light" model of the cosmic red-shift effect explaining [25].

On the other hand, according to an etheronic theory of

$$
\mathrm{F}_{\mathrm{s}}\left(\mathrm{m}_{\mathrm{p}}, \mathrm{v}\right)=\mathrm{k}_{\mathrm{h}} \cdot \mathrm{f}_{\mathrm{a}} \cdot \mathrm{m}_{\mathrm{p}} \cdot \rho_{\mathrm{s}}\left[(\mathrm{c}+\mathrm{v})^{2}-(\mathrm{c}-\mathrm{v})^{2}\right]=4 \mathrm{f}_{\mathrm{a}} \cdot \mathrm{k}_{\mathrm{h}} \mathrm{m}_{\mathrm{p}} \cdot \rho_{\mathrm{s}} \mathrm{c} \cdot \mathrm{v} \approx 6 \mathrm{f}_{\mathrm{a}}\left(\mathrm{m}_{\mathrm{p}} / \mathrm{m}_{\mathrm{h}}\right) \pi \cdot \mathrm{r}_{\mathrm{c}} \cdot \rho_{\mathrm{s}} \cdot \mathrm{v}_{\mathrm{s}} \cdot \mathrm{v}
$$

in which: $r_{c} \approx 1,8 \times 10^{-25} \mathrm{~m}$-the quanton's calibration radius (CGT, [9]), $v_{\mathrm{s}}=\eta_{\mathrm{v}} / \rho_{\mathrm{s}}$-the kinematic viscosity $\left(\eta_{\mathrm{v}}-\right.$ the dynamic viscosity), and $\mathrm{f}_{\mathrm{a}}<1$ - particle's form factor.

From the relation (27) it results the approximation:

$$
v_{\mathrm{s}}=(2 / 3) \cdot \mathrm{r}_{\mathrm{c}} \cdot \mathrm{c} \approx 3,6 \times 10^{-17} \mathrm{~m}^{2} / \mathrm{s} \text {. }
$$

Identifying- for the case of the interstellary space, the subquantum medium with the dark energy, we can take: $\rho_{\mathrm{s}}=1.2 \times 10^{-26} \mathrm{~kg} / \mathrm{m}^{3}$, resulting to the limit: $\mathrm{v}=\mathrm{c}$, that: $\mathrm{a}_{\mathrm{sM}}=\mathrm{F}_{\mathrm{s}}^{\mathrm{M}} / \mathrm{m}_{\mathrm{p}} \approx 3,31 \times 10^{-8} \mathrm{~N} / \mathrm{kg}$ - a value comparable to the gravitational acceleration generated by a mass of 1 ton at a distance of $1 \mathrm{~m}$, thus negligible on non-cosmic distances, compared to the terrestrial gravitational force, for example.

A consequence of the previous model of mass and charge increasing with the displacing speed is the conclusion that a very strong magnetic field with strong vortex tubes $\xi_{\mathrm{B}}$ which generates a magneto-gravitic potential $V_{\Gamma}$ of the form (27), may increase the mass, the charge and the magnetic moment of a particle.

It is interesting to observe that- according to the special Einsteinian relativity, composing the speeds according to Einstein's formula in the eqn. (27), it results a lack of drag gravitation of LeSage type [26], the drag force acting on a particle passing through the sub-quantum medium cannot be considered null. The compromise solution may be the including of a particle's form factor $f_{a}<1$ in the expression of the drag force generated at its passing through the fluid medium of the quantum vacuum, which- in the absence of the action of electrical or gravitational fields, is of Stokes type, because the laminar regime of the particle's displacing also at relativist speed $\mathrm{v} \rightarrow \mathrm{c}$, as in the case of the photon, [27].

The approximate value of this force can be equated by equivalenting the action of the quantum vacuum etheronic quanta with the action of some omnidirectional etheronic winds of the same mean impulse density: $p_{\mathrm{s}}=\rho_{\mathrm{s}} \cdot \mathrm{c}$ in a point $\mathrm{P}_{\mathrm{s}}$ in which the $\mathrm{m}_{\mathrm{p}}$-particle is stationary.

If the $\mathrm{m}_{\mathrm{p}^{-}}$particle will receive an impulse $\mathrm{m}_{\mathrm{p}} \mathrm{v}$ in a direction $x-x$ ', by the Galilean relativity we may obtain the expression of the drag force in accordance with eqn. (7), by the relation:

force at the particles's displacing through a quantum or subquantum medium with quanta having the light speed.

In consequence, it results that -by taking into account and the d'Alembert's paradox, the possibility of a real variation of mass with its speed may be theoretically sustained but according to the classical relation (9) which may contain also the effect of the drag force generated at the particle's passing through the quantum vacuum, relative to non-cosmic distances (smaller than the Terra's radius, $\mathrm{R}_{\mathrm{T}}$, for which the effect is small, $\eta_{\mathrm{s}} \rightarrow 0$ ).

Also, according to the explicative model, this possible real variation of particle's mass results only in the case of particle's displacement with the magnetic moment perpendicular to its impulse, in the case of the parallel orientation $(\mu / \mathrm{mv})$ the particle's mass variation with its speed resulting as formal and not real- according to the model.

The conclusion of a real mass increasing with the speed by quanta of the quantum vacuum was considered also by $\mathrm{M}$. Bukhari [28] in the photon's case, by a phenomenological model which argues that photon mass is an induced effect rendered in the form of vacuum potential. 
The particle's relativist impulse increasing with the speed, according to the relation: $\mathrm{p}\left(\mathrm{v}_{\mathrm{s}}\right)=\mathrm{p}_{\mathrm{o}} / \alpha$, (without speed reducing), imply the conclusion that the mass increasing $(\mathrm{m}=$ $\mathrm{m}_{\mathrm{o}} / \alpha$ ) is realized by the capture of quantum vacuum photons having the same speed $v_{\mathrm{s}}$ relative to the m-particle. In this case, we may suppose that the kinetic moment of an atomic electron increases with the atom's speed, according to the relation: $\mathrm{L}_{\mathrm{k}}\left(\mathrm{v}_{\mathrm{s}}\right)=\mathrm{m}_{\mathrm{e}}\left(\mathrm{v}_{\mathrm{s}}\right) \cdot \mathrm{v}_{\mathrm{e}} \cdot \mathrm{r}_{\mathrm{e}}=\mathrm{m}_{\mathrm{o}} \cdot \mathrm{v}_{\mathrm{o}} \cdot \mathrm{r}_{\mathrm{o}} / \alpha$.

The previous conclusions allows the use of the relation

(3) specific to a classic relativity instead the Einsteinian relation (4), avoiding the paradox of an infinite growth of particle's mass with its speed which- in accordance with the energy conservation law, imply the necessity of a high value of the quantum vacuum density which would generate an intense drag effect.

\section{Theoretical Implications}

1. A real phenomenological variation of both the mass and the charge with the particle's speed corresponding to the classical relation (9), in the case of the particle's displacement with the magnetic moment perpendicular to its impulse: $\mu_{p} \perp m_{p} v$, could explain the phenomenon of variation with the velocity of the time intervals measured by atomic clocks, without the Einsteinian relativity, in the following way:

Considering- for example, the case of a hydrogen atom of a M-body which moves at a relativistic speed $\mathrm{w} \rightarrow \mathrm{c}$, applying the relation (9) of the dynamic equilibrium equation to the case of the atomic electron's rotation to an orbit of $r_{0^{-}}$radius given by the kinetic momentum quantification relation: $\mathrm{m}_{\mathrm{e}}{ }^{0} \mathrm{v}_{0} \mathrm{r}_{0}=\mathrm{nh} / 2 \pi,\left(\mathrm{n}\left(\mathrm{r}_{0}\right)=1\right)$ and considering a real variation of particle's mass and charge, which imply also the variation of the electron's kinetic moment: $\mathrm{L}_{\mathrm{e}}(\mathrm{w})=\mathrm{m}_{\mathrm{e}}(\mathrm{w}) \cdot \mathrm{v}_{\mathrm{e}} \cdot \mathrm{r}_{\mathrm{e}}=$ $\mathrm{m}_{\mathrm{o}} \cdot \mathrm{v}_{\mathrm{o}} \cdot \mathrm{r}_{\mathrm{o}} / \alpha,\left(\alpha=\left(1-\mathrm{w}^{2} / 2 \mathrm{c}^{2}\right)\right)$, will results the relation:

$$
\frac{\mathrm{m}_{\mathrm{e}}^{0}}{\alpha} \frac{\mathrm{v}_{\mathrm{e}}^{2}}{\mathrm{r}_{\mathrm{e}}}=\frac{e_{0}^{2}}{\alpha^{2}} \frac{1}{4 \pi \varepsilon_{0} r_{e}^{2}} ; \Rightarrow \mathrm{m}_{\mathrm{e}}^{0} \mathrm{v}_{\mathrm{e}}^{2} \cdot r_{e}=\frac{e_{0}^{2}}{\alpha} \frac{1}{4 \pi \varepsilon_{0}}=\frac{1}{\alpha} \mathrm{m}_{\mathrm{e}}^{0} \mathrm{v}_{0}^{2} \cdot r_{o} ; \Rightarrow \mathrm{v}_{\mathrm{e}}=\frac{\mathrm{v}_{0}^{2} \cdot r_{o}}{\alpha \cdot \mathrm{v}_{\mathrm{o}} r_{o}}=\frac{\mathrm{v}_{0}}{\alpha}
$$

By the relation (29) results the conclusion that the atomic electron obtains a complete rotation around the nucleus within a shorter time interval $\tau$ :

$$
\tau(w)=\frac{2 \pi \cdot r_{e}}{\mathrm{v}_{\mathrm{e}}}=\frac{2 \pi \cdot \mathrm{v}_{\mathrm{o}} r_{o}}{\mathrm{v}_{\mathrm{e}}^{2}}=\tau_{0} \cdot \alpha^{2} \quad ; \quad \tau_{0}=\tau(0)=\frac{2 \pi \cdot r_{o}}{\mathrm{v}_{\mathrm{o}}}
$$

as a consequence of the fact that the atomic electron with increased mass and charge cover a portion $\delta l_{\mathrm{e}}=\delta \mathrm{l}_{0}$ of its orbital in a shorter time interval:

$$
\delta \tau_{\mathrm{e}}=\delta 1_{\mathrm{e}} / \mathrm{v}_{\mathrm{e}}=\left(\delta 1_{0} / \mathrm{v}_{\mathrm{o}}\right) \cdot \alpha=\delta \tau_{\mathrm{o}} \cdot \alpha .
$$

This effect was initially found by Larmor (1897) who concluded that the atomic electrons goes through parts corresponding to their trajectories in shorter time intervals for the ether-associated system, conform to the relation: $t=\alpha \cdot t_{0}$, but putting the effect on a length's contraction, [29].

For the values: $w=0.9 \mathrm{c} ; 0.95 \mathrm{c}$, for example, the relation (30) for the electron's rotation gives: $\tau(\mathrm{w})=0.354 \cdot \tau_{\mathrm{o}} ; 0.3 \cdot \tau_{\mathrm{o}}$, while the einsteinian relation gives: $\tau_{\mathrm{r}}(\mathrm{w})=\tau_{\mathrm{o}} / \gamma=0.4358 \cdot \tau_{\mathrm{o}}$; $0.31 \cdot \tau_{\mathrm{o}}$, (a relative close value).

The variation of the atomic electron's speed (29) in atoms of the Eart's reference system - considered as an etheronoquantonic energy storage system, is in accordance with the relation of the magnetic moment and with the vortexial atomic model (CGT, $[9,10])$ which explains the rotation of the atomic electrons around the nucleus without emission of radiation, by the nature of the $\Gamma_{\mu}$-etherono-quantonic vortex of the nuclear proton's magnetic moment, $\mu_{\mathrm{N}}$.

According to the previous theoretical considerations, it is also deduced that- in the absence of a quantum and subquantum medium, both the mass and the charge variations with the speed resulting from the relation (9) are canceled, but the conclusion of the speed-depending variation of the electric field intensity is maintained-according to the relation
(13), being explained in this way the fact that the Einsteinian relation (4) of the speed-depending variation of the particle's mass may be used without the concept of 'ether', by the postulate of the light speed constancy which- applied to the relations (11)-(13) of the electric field variation, gives:

$$
\mathrm{u}=\mathrm{c} \Rightarrow \mathrm{E}||\left(\mathrm{v}_{\mathrm{e}}\right)=\mathrm{E}||(0) / \gamma,\left(\gamma=1 / \sqrt{ }\left(1-\mathrm{v}^{2} / \mathrm{c}^{2}\right)\right),
$$

corresponding to an apparent mass variation (4).

2 . In the context of previous theoretical considerations, according to the relations (9) and (13), the photon appears as a "non-privileged" particle, with inertial rest- mass equal to half of the motion mass: $\mathrm{m}_{\mathrm{f}}^{0}=1 / 2 \mathrm{~m}_{\mathrm{f}}=1 / 2 \mathrm{hv} / \mathrm{c}^{2}$, in accordance with the phenomena caused predominantly by the photon's corpuscular nature, such as: the photoelectric effect, the transition of atomic electrons to a higher orbital or the generation of the light's pressure and so on.

In CGT, these effects were explained by a revised quantum-vortexial model of pseudo-scalar photon [10, 27], of Munera type, formed as a pair of two vectorial photons (vectons or vexons -in CGT) with opposed magnetic moments and spins and with super-dense centroid of its inertial mass, which generates a quantum vortex of the vexonic magnetic moment $\mu_{\mathrm{v}}$ and a spinorial mass $\mathrm{m}_{\mathrm{s}}{ }^{\mathrm{v}}$ of value equal to the inertial rest-mass, $[9,10,27]$ :

$\mathrm{m}_{\mathrm{s}}^{\mathrm{v}}=\mathrm{m}_{\mathrm{i}}^{\mathrm{v}}=1 / 2 \mathrm{~m}_{\mathrm{S}}=1 / 2 \mathrm{~m}_{\mathrm{f}}^{0}$, which gives a total mass- double than the inertial mass of the pseudo-scalar photon:

$$
\mathrm{m}_{\mathrm{f}}=2 \mathrm{x}\left(\mathrm{m}_{\mathrm{i}}^{\mathrm{v}}+\mathrm{m}_{\mathrm{s}}^{\mathrm{v}}\right)=2 \mathrm{x}\left(2 \mathrm{~m}_{\mathrm{i}}^{\mathrm{v}}\right)=\mathrm{m}_{\mathrm{f}}^{0} / \alpha=2 \mathrm{~m}_{\mathrm{f}}^{0} ;
$$

In this case, the pseudo-scalar photon has a kinetic energy: $\mathrm{E}_{\mathrm{f}}^{\mathrm{c}}=1 / 2 \mathrm{~m}_{\mathrm{f}} \mathrm{c}^{2}$ - according to relation (2) and a rotational (spinorial) energy: $\mathrm{E}_{\mathrm{f}}^{\mathrm{s}} \approx \mathrm{E}_{\mathrm{f}}^{\mathrm{c}}$, considering a rotation speed: $\mathrm{v}_{\omega}$ $=\omega \cdot \mathrm{r}=\mathrm{c}$ of the inertial $\mathrm{m}_{\mathrm{i}}{ }^{\mathrm{v}}$ - mass and of the spinorial $\mathrm{m}_{\mathrm{s}}^{\mathrm{v}}$ mass of the vectorial photons (which forms the pseudo-scalar photon), the translational kinetic energy of the spinorial $\mathrm{m}_{\mathrm{s}}^{\mathrm{v}}$ - 
mass of the vectorial photons being associated with the photon's electrical energy and the rotational energy of the $\mathrm{m}_{\mathrm{s}}{ }^{\mathrm{v}}$ - mass being associated with magnetic moment's energy of the vexon- according to the model [9]:

$$
E_{E}^{c}=E_{\mu}^{c} \Rightarrow \frac{\sum m_{h} c^{2}}{2}=\frac{m_{s}^{v} \cdot(\omega \cdot r)^{2}}{2}=\frac{m_{i}^{v} c^{2}}{2}=\frac{h v}{4} ; \quad h v=2\left(E_{E}^{c}+E_{\mu}^{c}\right), \quad \omega \cdot r=c
$$

According to eqns. (31), (32), the spinorial $\mathrm{m}_{\mathrm{s}}^{\mathrm{v}}$-mass of the vectorial photon is generated by the photon's displacing, which generates the relativist $\Gamma_{\mathrm{r}}^{\mathrm{v}}(\mathrm{v})$-vortex, but it may be generated also by the $\Gamma_{\mu}$-vortex of an external magnetic moment in which the photon is positioned, as in the case of the vectorial photons of the electron's quantum volume, which- in this way, explains its rest mass energy: $\mathrm{m}_{\mathrm{e}} \mathrm{c}^{2}-$ according to CGT $[9,10]$.

In this way, in a Galilean relativity, by eqn. (32) is explained the total energy of the pseudo-scalar photon: $\mathrm{E}_{\mathrm{f}}=\mathrm{m}_{\mathrm{f}} \mathrm{c}^{2}=\mathrm{hv}$ as real energy, transferable to other particles such as the electrons.

According to eqns. (31), (32), the spinorial $\mathrm{m}_{\mathrm{s}}^{\mathrm{v}}$-mass of the vectorial photon is generated by the photon's displacing, which generates the relativist $\Gamma_{\mathrm{r}}^{\mathrm{v}}(\mathrm{v})$-vortex, but it may be generated also by the $\Gamma_{\mu}$-vortex of an external magnetic moment in which the photon is positioned, as in the case of the vectorial photons of the electron's quantum volume, which- in this way, explains its rest mass energy: $\mathrm{m}_{\mathrm{e}} \mathrm{c}^{2}-$ according to CGT $[9,10]$.

In this way, in a Galilean relativity, by eqn. (32) is explained the total energy of the pseudo-scalar photon:

$\mathrm{E}_{\mathrm{f}}=\mathrm{m}_{\mathrm{f}} \mathrm{c}^{2}=\mathrm{hv}$ as real energy, transferable to other particles such as the electrons.

According to the model, the spinorial $\mathrm{m}_{\mathrm{s}}{ }^{\mathrm{v}}$ - mass of the vectorial photons is contained by the evanescent vortexial part, comprised in the volume of the Compton radius: $r_{\lambda}=\lambda / 2 \pi=$ $\mathrm{h} / 2 \pi \mathrm{m}_{\mathrm{s}}{ }^{\mathrm{v}} \mathrm{c}$ of the vexonic magnetic moment, given by the etherono-quantonic vortex $\Gamma_{\mu}^{\mathrm{v}}(\mathrm{r})$ that gives the magnetic energy: $\mathrm{E}_{\mathrm{H}} \sim 1 / 2 \mu_{0} \mathrm{H}^{2} \sim \Gamma_{\mathrm{H}}^{\mathrm{v}}$ and which - by confining and vortexing small quantum clusters (vectons- $\mathrm{m}_{\mathrm{v}}==3 \times 10^{10} \mathrm{~m}_{\mathrm{h}}=2.2 \times 10^{-40} \mathrm{~kg}$ [9]), produces the $\mathrm{m}_{\mathrm{s}}^{\mathrm{v}}$-spinning mass of the vectorial photons.

Also, the d'Alembert's paradox explains the relative lack of drag effect at the photon's displacing on non-cosmic distances and the variation with $\mathrm{r}^{-2}$ of the electrostatic E-field, (eq. (6)).

3 . The considering of a photon with rest mass involves- as is well known- the use of Maxwell-Proca equations and a Proca waveform relation [30]:

$$
\Delta \Phi-\frac{1}{c^{2}} \frac{\partial^{2} \Phi}{\partial t^{2}}-k^{2} \Phi=-\mathrm{g}_{\mathrm{q}} ; \Phi=(\phi, \mathrm{A}) ; \mathrm{g}_{\mathrm{q}}=\left(\frac{\rho_{\mathrm{q}}}{\varepsilon_{0}} ; \mu_{0} \cdot J_{q}\right) ; \quad \phi=\mathrm{V}_{\mathrm{q}}(\mathrm{r}, 0)=-\frac{q}{4 \pi \cdot \varepsilon_{0} r} e^{-k \cdot r} ; k=\frac{2 \pi}{\lambda}=\frac{m_{v}^{0} c}{\hbar}
$$

which gives an electrical potential $\varphi(\mathrm{r})$ and magnetic potential A (r) with a finite action radius: $l_{\mathrm{E}}=\mathrm{k}^{-1}$, the electric potential $\mathrm{V}_{\mathrm{q}}$ generated by a quasi-punctiform charge $\mathrm{q}$ having- for the stationary case, an expression of Yukawa type.

In the context in which- for the electric field $\mathrm{E}=-\nabla \mathrm{V}_{\mathrm{q}}$, we consider as explanatory the relation (6) of the CGT- specific to a spheric-symmetrical quanta distribution of the q-charge,

$$
E_{q}(r)=\mathrm{k}_{1} \rho_{\mathrm{v}} \mathrm{v}_{\mathrm{v}}^{2}=\mathrm{k}_{1} n_{v}^{0} m_{\mathrm{v}} \mathrm{v}_{\mathrm{v}}^{2}=\mathrm{E}_{\mathrm{q}}^{0} \frac{a^{2}}{r^{2}} e^{-k \cdot r}(1-\mathrm{k} \cdot \mathrm{r})
$$

This phenomenon, in the pseudo-scalar photon's case, is known in astrophysics as "radiation aging" effect and is considered to be a partial cause of the red-shift effect of the radiation frequency modifying, according to some theories [31], but other authors argued that the radiation aging model is not fitted with the astrophysical observations, if it is considered the main cause of the red-shift effect [25].

In the case of vectorial photons of the electric E -field, it appears as a photon energy attenuation effect which modify also the E-field value.

But- according to eqn. (27) and to d'Alembert paradox, this effect results as negligible on non-cosmic distances, with priority for the vectorial photons whose spin is parallel or antiparallel with the impulse - according to the pseudoscalar charge model of CGT $[9,10]$, because that this orientation of the spin generates a minimal drag force at the advancement but considering vectorial photons with rest mass, as field quanta, the only characteristic of the quanta flux which can produce an exponential decrease with the factor $\mathrm{e}^{\mathrm{kr}}$ of the $\mathrm{V}_{\mathrm{q}^{-}}$ potential and of the electric E- field - in addition to the decrease with $r^{2}$, is the decrease of the field quanta's energy as a result of the drag force at the quanta advancement through the sub-quantum medium, $\mathrm{F}_{\mathrm{rv}}$ :

$\mathrm{k}=\frac{2 \pi}{\lambda_{\mathrm{f}}}=\frac{1}{l_{E}}=\frac{m_{\mathrm{v}}^{0} c}{\hbar} ; \rho_{\mathrm{v}} \propto r^{-2} ; \varepsilon=\mathrm{m}_{\mathrm{v}} \mathrm{v}_{\mathrm{v}}^{2} \propto \mathrm{e}^{-\mathrm{k} \cdot \mathrm{r}}$

through the sub-quantum medium, by considering a fusiform (possibly- helical) form of the super-dense centroid that ensures the stability of the vortexial structure of the vectorial photon - according to the model $[9,10]$.

This explains the result of Coulomb's law verification experiments that indicates a rest mass of the photons which mediates the electric interaction: $\mathrm{m}_{\mathrm{f}} \leq 10^{-14} \mathrm{eV} / \mathrm{c}^{2},\left(1.7 \times 10^{-}\right.$ ${ }^{50} \mathrm{Kg}$ [3]) -value that corresponds -by the relations (27), (34) and (35), to a variation of the photon's speed of the form: $\mathrm{v}_{\mathrm{v}}$ $=\mathrm{c} \cdot \mathrm{e}^{-\mathrm{k} \cdot \mathrm{r}}$ with: $\mathrm{k}=\mathrm{m}_{\mathrm{f}} \mathrm{c} / \mathrm{h} \approx 4.84 \times 10^{-8} \mathrm{~m}^{-1},\left(\mathrm{l}_{\mathrm{E}}=\mathrm{k}^{-1}=\right.$ $\left.2 \times 10^{7} \mathrm{~m}\right)$.

Since $l_{\mathrm{E}}=1 / \mathrm{k}$ represents the Compton radius of electrostatic force's action, the compatibility of the previous mentioned experimental result with a photonic rest mass corresponding to the classical relativistic relation (3) and with the relations (33) - (35), may result by considering a 
value: $\mathrm{k}_{\mathrm{a}}=\mathrm{f}_{\mathrm{a}} \cdot \mathrm{k}=\mathrm{f}_{\mathrm{a}} \cdot\left(\mathrm{m}_{\mathrm{v}}{ }^{0} \mathrm{c} / \mathrm{h}\right)$ with: $\mathrm{m}_{\mathrm{v}}{ }^{0} \approx 10^{-40} \mathrm{~kg}$ (the vecton's rest mass, $[9,10])$ with $\mathrm{f}_{\mathrm{a}} \approx 10^{-10}$ - form factor, taking into account the d'Alembert's paradox.

The substitution in the relation (33) - (35) of the constant $\mathrm{k}$ with the constant $\mathrm{k}_{\mathrm{a}}$ allows to consider only the variation with $r^{-2}$, specific to the Coulombian potential, of the electric field intensity, without the hypothesis of a null rest mass of the photon.

In this context, the hypothesis of the existence of a Higgs-type boson field generating the elementary particle's rest mass becomes unnecessary, the equivalent of the so-called "gluonic shell" of the quarks (surrounding the quark's current mass) being a shell of "frozen photons" -in CGT, with an rest energy $\varepsilon_{0}=$ $1 / 2 \mathrm{hv}=\mathrm{m}_{\mathrm{f}}^{0} \mathrm{c}^{2}$ given by the vortexial kinetics of its mass $[9,27]$.

In the case of a fermion, like the electron, the hypothesis of the magnetic moment increasing with the speed, according to eqn. (24), implying the conclusion of the spin' increasing, because that -in a neo-classical, pre-quantum model of particle, the spin $\mathrm{S}$ is given by a spinorial mass equal with the inertial mass: $\mathrm{m}_{\mathrm{s}}=\mathrm{m}_{\mathrm{i}},[9,10]$. It results that - if we take into account an increasing of the inertial mass, we must consider a speed-depending increasing also for the spinorial mass.

The non-participation of the spinorial mass to the inertial mass is explained in CGT by its evanescent character, given by mass quanta weakly interacted with the inertial mass.

4. Another indirect consequence, resulting from the consideration of a non-zero kinematic viscosity of the subquantum medium: $v_{\mathrm{s}}=\eta_{\mathrm{v}} / \rho_{\mathrm{s}} \approx 3,6 \times 10^{-17} \mathrm{~m}^{2} / \mathrm{s}$, is the conclusion that in an etherono-quantonic medium with increased density and relatively high dynamic viscosity, such as that of a super-cooled atomic system, the drag force $F_{s}$ which- to a crystal explains its refractive index $n$ by the decreasing of the photons speed: $\mathrm{n}_{\mathrm{r}}=\mathrm{c} / \mathrm{v}_{\mathrm{f}}$, may produce a high deceleration of the photon's displacement, phenomenon observed experimentally by a laser beam crossing through a Bose-Einstein condensate [1].

\section{Conclusions}

By an electric charge model of static type with spherical distribution of field quanta and by the Galilean relativity there are re-obtained the Lorentz's expressions of the longitudinal and transversal speed-depending mass of a charged particle accelerated by a quanta flux pressure, as apparent effect generated by a real decreasing of value of the longitudinal $\mathrm{E}_{\mathrm{L}}$ electric field, with $\mathrm{E}_{\mathrm{L}} \sim \gamma^{-3}$-at non-null angles: $\theta(\mathrm{r}, \mathrm{p})>0$, (between $\mathrm{r}(\delta \mathrm{Q}-\mathrm{q})$ and $\left.\mathrm{p}=\mathrm{m}_{\mathrm{q}} \mathrm{v}_{\mathrm{q}}\right)$ and with: $\mathrm{E}_{\mathrm{l}}\left(\mathrm{v}_{\mathrm{q}}, \theta=0\right)=\mathrm{E}_{1}^{0} \cdot \gamma^{-1}\left(1-\mathrm{v}_{\mathrm{q}} / \mathrm{c}\right)$ for $\theta(\mathrm{r}, \mathrm{p}) \approx 0$, but also of the transversal $\mathrm{E}_{\mathrm{T}}$-electric field and $\mathrm{B}_{\mathrm{T}}-$ magnetic field:

$$
\mathrm{E}_{\mathrm{T}} \sim \gamma^{-1}, \mathrm{~B}_{\mathrm{T}} \sim \gamma^{-1},\left(\gamma=1 / \sqrt{ }\left(1-\mathrm{v}^{2} / \mathrm{c}^{2}\right)\right) .
$$

In this case, the paradoxical Einsteinian conclusion [32] of a real mass increasing with the speed, may be avoided by considering a classical expression of both mass and charge variation, in the form: $\mathrm{m}=\mathrm{m}_{\mathrm{o}} / \alpha^{;} \mathrm{q}=\mathrm{q}_{\mathrm{o}} / \alpha$, with $\alpha=\left(1-\mathrm{v}^{2} / 2 \mathrm{c}^{2}\right)$.

By the explicative model is re-obtained also the general form of the Doppler-Fizeau effect, but in a Galilean relativity.
Also, the invariance of the Lorentz force expression indicates a similar relativist variation of the magnetic field induction as those of the transversal electric field: $\mathrm{B} \sim \gamma^{-1}$, which explains the experimental result of the Kaufmann-Bucherer experiments.

It results also that the obtained relations of $E_{L}, E_{1}$ and $E_{T}$, depending of field quanta flux, may be extrapolated- by eqns. (16) and (18), for the expression of a laser-like radiation pressure over a moving body.

It is argued also that a real mass and charge increasing with the $\alpha$-factor imply a significant value of the etheronoquantonic density of the quantum vacuum and may be generated only by a relativist etherono-quantonic vortex $\Gamma_{\mathrm{r}}(\mathrm{v})$ which is added to the similar vortex $\Gamma_{\mu}(0)$ of the particle's magnetic moment which- in this way, is increased with the speed, $\left(\mu(v)=\mu_{0} / \alpha\right)$, if the particle's spin is rectangular to its impulse, the particle's mass increasing resulting by quanta confining in the particle's self- potential vortexially generated.

According to the explicative model, the photon results as a 'non-privileged' particle, with an inertial rest-mass $m_{i}$ - half of its relativist mass $\mathrm{m}_{\mathrm{f}}(\mathrm{c})$ - given by the addition to its inertial rest-mass of its spinorial mass $m_{s}$, whose total energy is explained as sum of its translation energy and its rotational energy- associated with the magnetic moment's vortexial energy of the paired vectorial photons which forms a photonic pseudo-scalar quanta, according to a dual (wavecorpuscle) model of photon [27].

The relative lack of drag effect at the photon's displacing on non-cosmic distances may also be explained, by the d'Alembert's paradox, without the rejection of the ether's existence conclusion.

This possibility permits to maintain the conclusion of longrange action of the coulombian field, by considering an Yukawatype electric potential, specific to a Proca-type field equation, of the form: $V(r)=V_{0} \cdot(a / r) \cdot e^{-k \cdot r}$ with: $k=f_{a} \cdot m_{f} c / h ; f_{a}<<1-$ form factor, taking into account d'Alembert's paradox.

The explanatory model of mass and charge increasing with the particle's speed permits the conclusion that a very strong magnetic field with strong vortex tubes $\xi_{\mathrm{B}}$ which generates a magneto-gravitic potential, of quanta impulse density gradient, may increase the mass, the charge and the magnetic moment of a particle which is placed in the magnetic field.

From the correspondence with the classical relativity explaining, it results also as plausible a pulsatory model of elementary charge, 'e, which- in accordance with the matterenergy conservation law, suppose a conversion mechanism of light photons of $2.7 \mathrm{~K}$ background radiation into vectorial photons ("vectons"- $\mathrm{m}_{\mathrm{v}} \geq 2.3 \times 10^{-40} \mathrm{~kg}[9,10]$ ) in a pulsatory way, consisting in the acquiring of a critical quantity $\delta m_{f}$ of background radiation photons: $\mathrm{m}_{\mathrm{f}}=2 \mathrm{~m}_{\mathrm{v}}$, in the time period $\tau_{0}$ and the vortexial conversion (by the $\Gamma_{\mu}$-vortex of the $\mu_{\mathrm{e}}-$ magnetic moment) into $\mathrm{n}_{\mathrm{f}}=\delta \mathrm{m}_{\mathrm{f}} / \mathrm{m}_{\mathrm{v}}$ vectons which are simultaneously emitted after the time period $\tau_{0}$, this process being favored by the zeroth vibrations of the e-charge $\left({ }^{1} / 2 \mathrm{hv}_{0}\right)$ which generates periodical inflation of the particle's quantum volume which becomes in this way, periodically, penetrable 
to relativist photons action.

It is also plausible- according to this model of e-charge, that the quantum volume of the acquired background radiation photons is decreased at their incorporation into the quantum volume of the electron- as consequence of the increasing of local dynamic and static quantum pressure, at the vectons' releasing the process being inversed.

It results also that the hypothesis of a heavy boson which gives rest mass to other particles (the Higgs boson) is not strictly necessary, from phenomenological point of view, in the sense that it may be only a particular case, the strong interactions between particles with mass excess (the total mass of the resulted particles- greater than those of the particles entered in reaction) being explained- in this case, by the participation of quantum vacuum bosons with specific mass and with structure of quark-antiquark pairs.

\section{References}

[1] Harris, S. E., Hau, L. V., "Nonlinear Optics at Low Light Levels", Phys. Rev. Lett. 82, 4611, (1999).

[2] Klaers, J., Schmitt, J., Vewinger, F., Weitz, M., "Bose-Einstein condensation of photons in an optical microcavity", Nature, 468, pp. 546-548, (2010).

[3] Williams, E., Faller, J.; Hill, H. "New Experimental Test of Coulomb's Law: A Laboratory Upper Limit on the Photon Rest Mass", Phys. Rev. Letters, 26, (12): 721, (1971).

[4] Quigg, C., Shrock, R., "Gedanken Worlds without Higgs: QCD-Induced Electroweak Symmetry Breaking", arXiv: 0901.3958v2 [hep-ph], (2009).

[5] Manor, E. P., "Gravity, Not Mass Increases with Velocity", Journ. of Modern Physics, 6, pp. 1407-1411, (2015).

[6] Ajay Sharma, "The Various Equations of Variation of Mass with Velocity", Indian Journal of Technology, 10, Issue 21, June (2017).

[7] Zhao Gong-Bo, Raveri M. et al., "Dynamical dark energy in light of the latest Observations", Nature Astronomy, 1 (9), 627, (2017).

[8] Kaufmann, W., "Über die elektromagnetische Masse des Elektrons", Physikalische Zeitschrift, 4 (1b): pp. 54-57, (1902).

[9] Arghirescu, M., "The Cold Genesis of Matter and Fields", Science Publishing Group, (2015).

[10] Arghirescu, M., "A Quasi-Unitary Pre-Quantum theory of Particles and Fields and some Theoretical Implications", IJHEP, july, pp. 80-103, (2015).

[11] Einstein, Albert, "Zur Elektrodynamik bewegter Körper, Annalen der Physik 17, 1, pp. 891- 921, (1905).

[12] Lorentz, H. A., Amsterdam Proceedings, 12, (1904).

[13] Janssen, M., Mecklenburg, M., "Electromagnetic Models of the Electron and the Transition from Classical to Relativistic Mechanics", (2004); http://philsciarchive.pitt.edu/id/eprint/1990.
[14] Brown, B. C.; Masek, G. E. et al., "Experimental Comparison of the Velocities of $\mathrm{eV}$ (Visible) and GeV Electromagnetic Radiation" Phys. Rev. Letters, 30 (16): pp. 763-766, (1973).

[15] Guiragossián, Z. G. T., Rothbart, G. B. et al., "Relative Velocity Measurements of Electrons and Gamma Rays at 15 GeV", Phys. Rev. Letters, 34 (6): pp. 335-338, (1974).

[16] Pierseaux, Y., "Poincare's relativistic Doppler- Fizeau formula", arXiv: physics/0605069v1 [physics.class-ph], (2006).

[17] Tai L. Chow, "Electromagnetic theory", Sudbury MA: Jones and Bartlett, Chapter 10.21; pp. 402-403, (2006).

[18] Bucherer, A. H., 'Die experimentelle Bestätigung des Relativitätsprinzips', Annalen der Physik. 333 (3): pp. 513536, (1909).

[19] Rogers, M. M.; et al., 'A Determination of the Masses and Velocities of Three Radium B Beta- Particles', Physical Review, 57: pp. 379-383, (1940).

[20] Castillo, A. M., "A new light in physics", www.createspace.com, (2017).

[21] Laghari, A. A., 'Asif's Equation of Charge Variation and Special Relativity', IOSR Journal of Applied Physics, Vol. 4, Issue 3, pp. 01-04, (2013).

[22] Constantinescu, P., "Hierarchized Systems", Ed. Acad. R. S. R., Bucharest, 106, (1986).

[23] Saint-Venant, A., "Mémoire sur la théorie de la résistance des fluides. Solution du paradoxe proposé à ce sujet par d'Alembert aux géomètres. Comparaison de la théorie aux expériences," Comptes Rendus des Séances de l'Académie des Sciences, 24: pp. 243-246, (1847).

[24] Sbitnev, V. I., "Physical vacuum is a special superfluid medium", arXiv: 1501.06763v4.

[25] Geller, M. J., Peebles, P. J. E., "Test of the expanding universe postulate", The Astrophysical Journal, 174: 15 May, pp. 1-5, (1972).

[26] Georges L. Lesage, "Lucrèce newtonien", Mémoires de l'Académie royale des sciences et belles-lettres de Berlin pour 1782, Berlin, pp. 1-28, (1784).

[27] Arghirescu, M., "A Revised Model of Photon Resulted by an Etherono-Quantonic Theory of Fields", Open Access Library Journal, 2: e1920, (2015).

[28] Bukhari, M. H. S., "A phenomenological model for photon mass generation in vacuo", International Journal of Physical Sciences, 9 (4), pp. 48-53, (2014).

[29] Larmor, J., "A dynamical theory of the electric and luminiferous medium.- Part III, relations with material media', Phil. Trans. R. Soc. Lond. A 190, pp. 205-300.

[30] (1897); http://rsta.royalsocietypublishing.org/ Tu, L-C., Luo, J., Gillies, G. T., "The mass of the photon", Rep. Prog. Phys. 68, pp. 77-130, (2005).

[31] Zwicky, F., "On the Red Shift of Spectral Lines through Interstellar Space”, PNAS 15: pp. 773-779, (1929).

[32] Wang, L. J., “A critique on Einstein's mass-energy relationship and Heisenberg's uncertainty principle", Physics Essays 30, 1 (2017). 